\title{
Comparison of Volumetric Fat Suppressed Proton Density and Standard Routine MR Sequences in the Evaluation of Meniscal Tears
}

\author{
HAZEM H. SOLIMAN, M.D. and MAGED A. HAWANA, M.D. \\ The Department of Radiology, Faculty of Medicine, Cairo University, Egypt
}

\begin{abstract}
Background: The knee joint is the most common joint liable for injuries with knee MRI being the most preferred imaging modality for the assessment of knee pain. The commonest indications are the evaluation of the meniscal lesions, and ligamentous injuries.
\end{abstract}

Arthroscopy is considered the gold standard tool for the assessment of knee derangement, although knee MR examination has shown significant influence upon the clinicians' management decisions.

Current standard two dimensional MR protocol is time consuming with limitations of these sequences that have to be considered.

Volumetric fat suppressed proton density sequence is used with the advantage of faster acquisition in comparison with standard routine sequences.

The volumetric PD FS sequence can demonstrate similar diagnostic results to that of the standard three planes sequences in the assessment of internal knee derangement especially as regards meniscal injuries.

Aim of Study: To assess the diagnostic accuracy of volumetric fat suppressed proton density sequence versus standard two dimensional (2D) sequences at 1.5T MR in the evaluation of meniscal tears.

Patients and Methods: The study included 81 patients presenting with suspected post traumatic meniscal injury. Patients were referred to the Kasr Al-Aini Hospital Radiology Department between February 2018 and January 2019 after full clinical examination to evaluate their knee joints using MRI.

Results: In our study the results showed typical concordance between standard two dimensional (2D) three planes sequences and volumetric fat suppressed proton density sequence in the detection of medial and lateral meniscal tears after using arthroscopy as the gold standard. Both sequences showed sensitivity $=95.92 \%$, specificity $=100 \%$, Positive Predictive Value (PPV) $=100 \%$, Negative Predictive Value (NPV) $=94.12 \%$ and accuracy $=97.53 \%$ for Medial Meniscal $(\mathrm{MM})$ tears as well as sensitivity $=75 \%$, specificity $=100 \% \mathrm{PPV}=$

Correspondence to: Dr. Hazem H. Soliman, The Department of Radiology, Faculty of Medicine, Cairo University, Egypt
$100 \%, \mathrm{NPV}=94.2 \%$ and accuracy $=95.06 \%$ for Lateral Meniscal (LM) tears.

Conclusion: The volumetric fat suppressed proton density sequence had shorter acquisition time and delivered similar high diagnostic accuracy to the standard 2D sequences in diagnosing meniscal tears.

Key Words: MRI-Knee joint-Meniscal-Tears.

\section{Introduction}

MRI has been established as an effective technique to evaluate meniscal tears of the knee [1]. Conventional spin-echo MR images have traditionally been used with reported sensitivities and specificities of $90-95 \%$ for the detection of meniscal tears [2].

Current standard two dimensional (2D) MR sequences had some limitations including increased gaps between sections which can hide joint abnormalities due to partial volume effect [3].

Volumetric fat suppressed proton density sequences are used with the advantage of faster acquisition in comparison with conventional three plans sequences [4].

Recent MRI machines allow acquisition of Volumetric fat suppressed proton density with the advantage to acquire Multiplanar Reformating (MPRs) because of the obtained isotropic images [6].

The three dimensional volumetric Proton Density Fat Suppressed sequence (3D-PDFS) is more accurate in having thinner slices than that of the orthogonal three planes images with good diagnostic accuracy, thus comparing the two techniques is valuable in revealing their efficiency in meniscal injury detection [2]. 
Other advantages of the 3D sequence include decreased acquisition time which is about 7 minutes for the 3D sequence compared to 13 minutes for the standard sequences. The reduction in scan time has the potential to improve patient experience and also to reduce costs with a time saving [9].

Optimized 3D-PDFS produces higher signal and contrast compared to conventional routine sequences, particularly for fluid and cartilage, leading to improved diagnostic accuracy, particularly in problematic lesions [10].

One disadvantage of a 3D sequence is that the whole sequence must be repeated if there is excessive movement artifact [9] .

\section{Patients and Methods}

The study was allowed by the Hospital Ethical Committee. The study included 81 patients presenting with suspected post traumatic meniscal injury the patients' ages ranged from 15-60 years (mean age 37), 51 males and 30 females.

Patients were referred to the Kasr Al-Aini Hospital Radiology Department between February 2018 and January 2019 after full clinical examination (including Mac Murray and Thessaly tests) to evaluate their knee joints using MRI.

MRI was performed using Philips scannres Achieva or Intera $(1.5 \mathrm{~T})$ by knee coil in all cases.
The standard knee protocol (Sagittal T 1 WIs, Sagittal T2 WIs, Sagittal PD WIs, Axial T2WIs and Coronal T2 FFE WIs) was designated Protocol $\mathrm{A}$, while the volumetric PD-FS imaging was designated Protocol B (Table 1).

\section{Inclusion criteria:}

Post traumatic knee pain showing signs of suspected meniscal injury with positive Mac Murray and Thessaly tests.

\section{Exclusion criteria:}

Patients with absolute contraindications to MR examination as cardiac pace maker, aneurysmal clipping and claustrophobia.

\section{Interpretation and data analysis:}

Two musculoskeletal radiology fellows, were blinded from arthroscopy and the radiology reports and retrospectively assessed both MR images.

Both menisci were assessed along their length and were reported by being positive or negative for meniscal tears.

Reformatting the Volumetric fat suppressed proton density with $0.6 \mathrm{~mm}$ section thickness in the sagittal and coronal planes were obtained semi automatically. Arthroscopy was considered as gold standard tool.

Table (1): Protocol A and B of MR imaging.

\begin{tabular}{lccccccc}
\hline Parameters & $\begin{array}{c}\text { Sagittal } \\
\text { T1 }\end{array}$ & $\begin{array}{c}\text { Sagittal } \\
\text { T2 }\end{array}$ & $\begin{array}{c}\text { Sagittal } \\
\text { STIR }\end{array}$ & $\begin{array}{c}\text { Sagittal } \\
\text { PD }\end{array}$ & $\begin{array}{c}\text { Coronal } \\
\text { T2 FFE }\end{array}$ & $\begin{array}{c}\text { Axial } \\
\text { T2 }\end{array}$ & $\begin{array}{c}\text { Saggital } \\
\text { PDFS }\end{array}$ \\
\hline TR & 600 & 3600 & 2400 & 1620 & 380 & 3600 & 1600 \\
TE & 17 & 100 & 60 & 30 & 13 & 100 & 33 \\
FOV: & & & & & & & \\
Anterior/posterior & 30 & 30 & 30 & 30 & 30 & 20 & 40 \\
Right/left & 35 & 35 & 50 & 35 & 50 & 60 & 30 \\
Feet/head & 50 & 50 & 20 & 50 & 20 & 40 & 50 \\
\hline
\end{tabular}

\section{Results}

\section{Protocol A MRI findings:}

2D MRI detected Medial Meniscus (MM) injury in 47 knee joints while Arthroscopy detected it in 49 knee joints, both modalities agreed in 47 patients and 2 lesions missed by MRI; sensitivity $=95.92 \%$, specificity $=100 \%$, Positive Predictive Value (PPV) $=100 \%$, Negative Predictive Value $=94.12 \%$ (NPV) and accuracy $=97.53 \%$.
2D MRI detected Lateral Meniscus (LM) injury in 12 knee joints while Arthroscopy detected it in 16 knee joints, both modalities agreed in 12 patient and 4 lesions missed by MRI. Sensitivity $=75 \%$, specificity $=100 \% \mathrm{PPV}=100 \% \mathrm{NPV}=94.2 \%$ and accuracy $=95.06 \%$.

\section{Protocol B findings:}

Volumetric MRI detected MM injury in in 47 knee joints while Arthroscopy detected it in 49 
knee joints, both modalities agreed in 47 patients and 2 lesions missed by MRI, sensitivity $=95.92 \%$ specificity $=100 \%, \mathrm{PPV}=100 \%, \mathrm{NPV}=94.12 \%$ and accuracy $=97.53 \%$.

Volumetric MRI detected LM injury in 12 knee joints while Arthroscopy detected it in 16 knee joints, both modalities agreed in 12 patient and 4 lesions missed by MRI. Sensitivity $=75 \%$ specificity $=100 \% \mathrm{PPV}=100 \% \mathrm{NPV}=94.2 \%$ accuracy $=$ $95.06 \%$.

Evaluation of the 3D volumetric PD FS sequence showed concordant diagnostic accuracy in the detection of medial and lateral meniscal tears in comparison with the standard sequences.
(A)

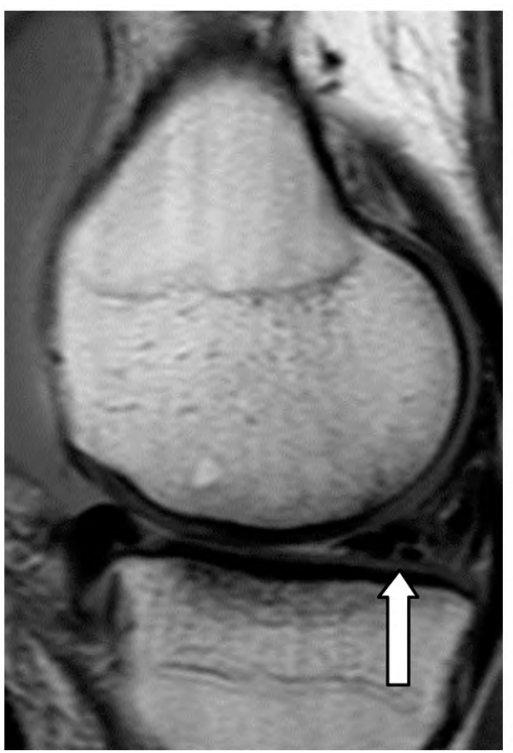

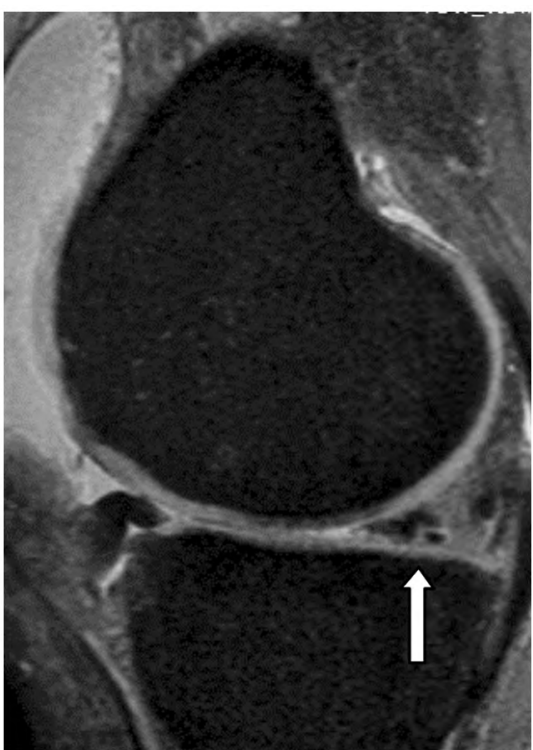

(B)

Fig. (1): 23 years old male patient presented by acute LT knee joint pain after trauma. (A \& B) sagittal standard 2D PDWI and 3D PD-FS images showing medial meniscus inferior surface tear (white arrows).

(A)

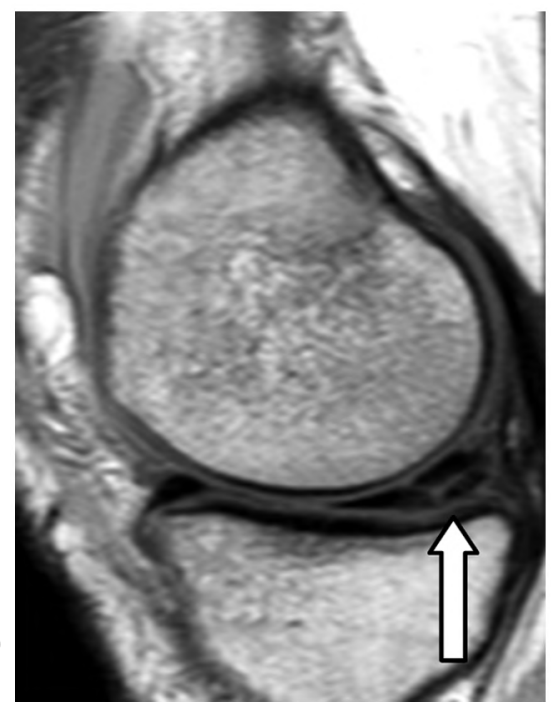

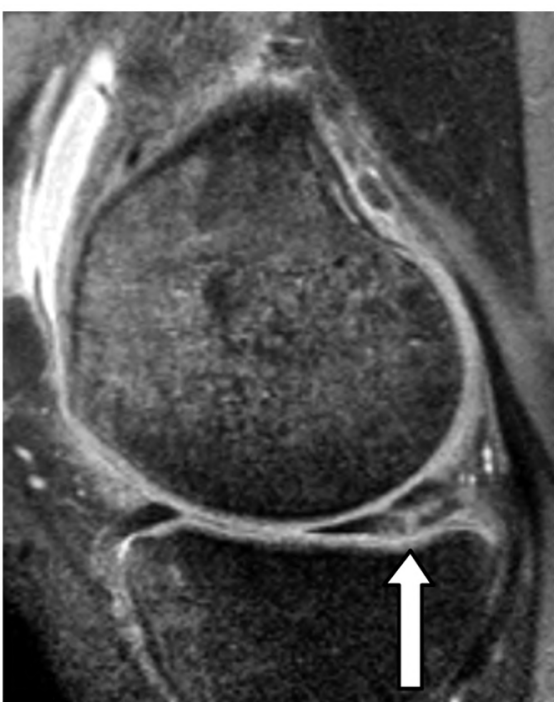

(B)

Fig. (2): 17 years old female patient presented by acute right knee joint pain after trauma. (A \& B) sagittal standard 2D PDWI and 3D PD-FS images showing medial meniscus superior and inferior surface tear (white arrows). 
(A)
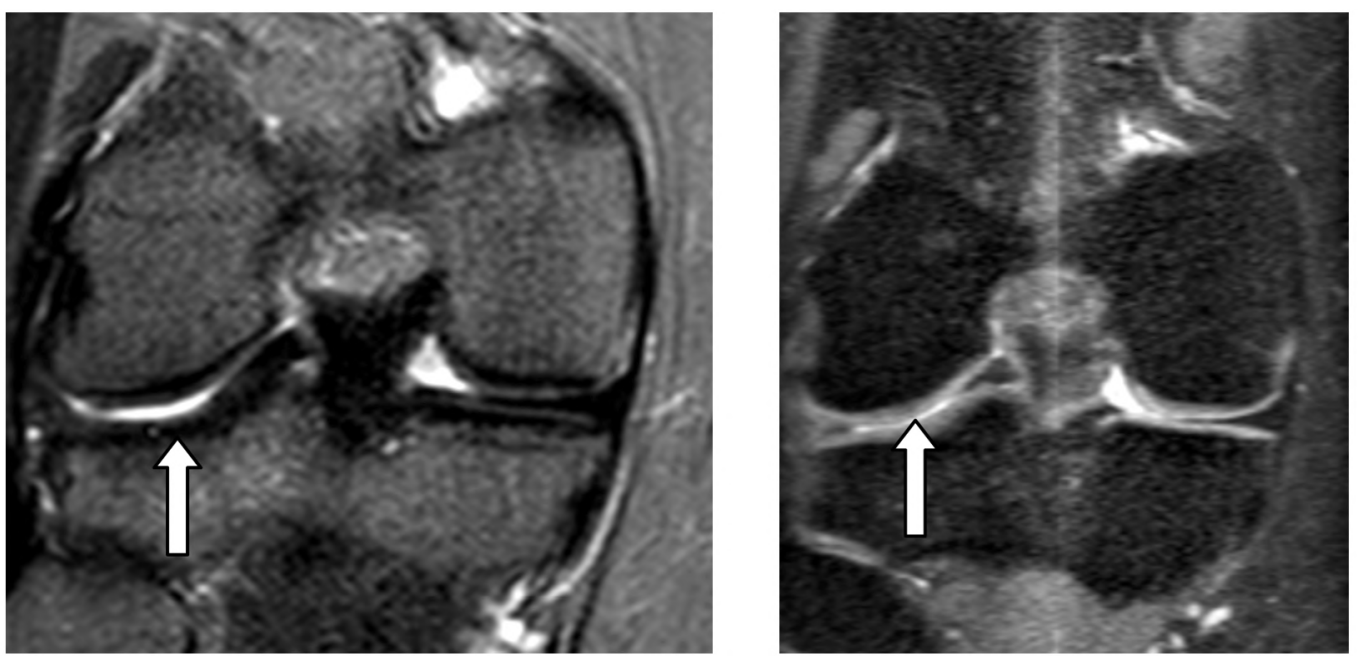

(B)

Fig. (3): 28 years old male patient presented by left knee joint pain after trauma. (A \& B) Coronal standard 2D STIR WI and Reformatted coronal 3D PD-FS images showing bucket handle tear of the lateral meniscus tear (white arrows).

(A)

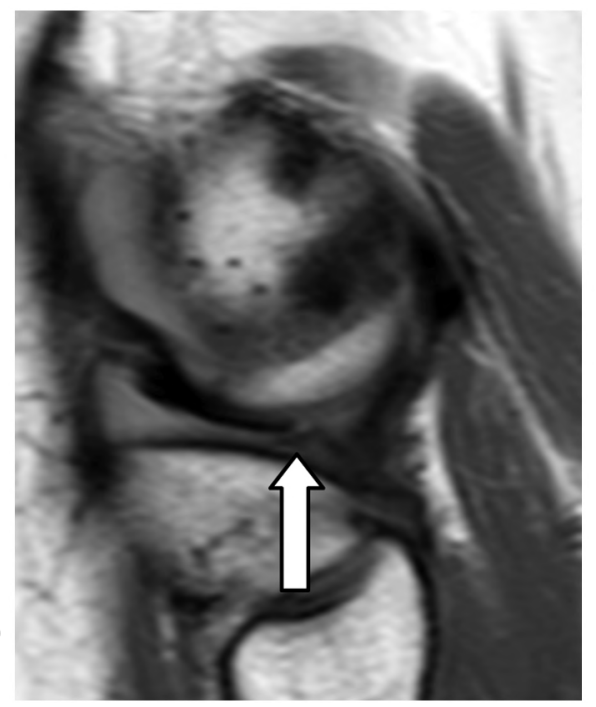

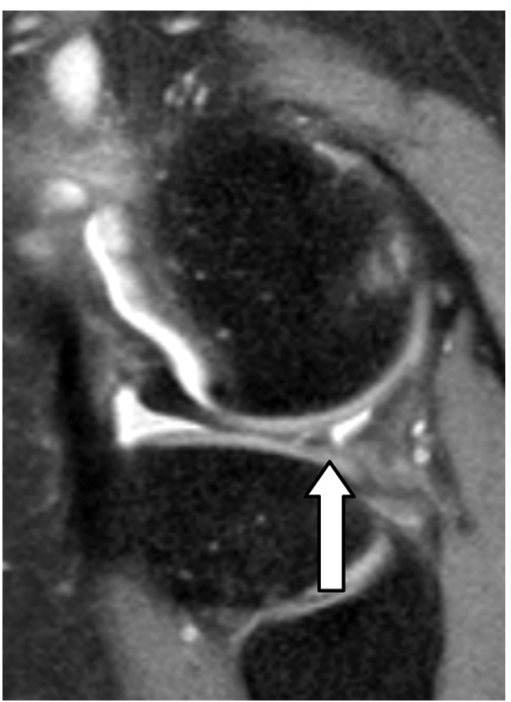

(B)

Fig. (4): 22 years old male patient presented by left knee joint pain after trauma. (A \& B) Sagittal standard 2D PDWI and 3D PD-FS images showing macerated lateral meniscus body and posterior horn/complex tear (white arrows).

\section{Discussion}

Yoon and his colleagues [7], demonstrated that volumetric PD FS sequence had similar diagnostic performance to a conventional 2D imaging protocol and reported that volumetric PD FS sensitivity, specificity, and accuracy of $100 \%, 82 \%$, and $90 \%$, respectively, for the diagnosis of MM tears; and $83 \%, 83 \%$, and $83 \%$, respectively, for lateral meniscus tears.

In our study the results showed sensitivity= $95.92 \%$, specificity $=100 \%$, positive predictive value $=100 \%$, negative predictive value $=94.12 \%$ and accuracy $=97.53 \%$ for the MM tears in comparison to sensitivity $=75 \%$ specificity $=100 \% \mathrm{PPV}$
$=100 \% \mathrm{NPV}=94.2 \%$ and accuracy $=95.06 \%$ for $\mathrm{LM}$ tears.

Daves and his colleagues were concordant with us in that volumetric imaging had high sensitivity and specificity for assessment of MM injuries compared to the standard MRI images [2].

In addition Chen and his colleagues agreed with us that volumetric PD FS sequence had similar diagnostic accuracy in the detection of the meniscal tears compared with routine MR sequences with accuracy (93\%) for MM and LM tears [1].

Recently, Lee and his colleagues stated that volumetric sequences had a similar accuracy $(95 \%)$ 
for evaluating both MM and LM tears compared with a standard MRI images [5] . Our study also demonstrated no significant difference in the diagnostic accuracy of 3D-PDFS in the detection of both medial and lateral meniscal tears.

A small study by $\mathrm{Ai}$ and his colleagues also demonstrated that the $3 \mathrm{D}$ sequence at $1.5 \mathrm{~T}$ had similar or superior sensitivity, specificity and accuracy to a conventional $2 \mathrm{D}$ imaging protocol [9].

Pass and his colleagues' evaluation of the 3D FSE PD FS sequence was in agreement with our results. Their study showed overall concordant accuracy in both medial and lateral meniscal tears in comparison with the standard sequences: $93.7 \%$ lateral and $90.9 \%$ medial menisci [8].

Kudo and his collagaues concluded similar findings to our study in that the routine use of reformatted thin-section 3D MRI using 3D PDFS sequences increases the diagnostic capability and confidence in assessment of meniscal tears. Thirtyeight meniscal lesions were surgically detected with excellent interobserver agreements (kappa= 0.91-0.98) seen using both MRI protocols, with a slightly better tendency in the reformatted 3D MRI protocol [10].

Nancy and her colleagues studied 432 menisci and concluded that sensitivities of volumetric PDFS and fast spin-echo imaging were equal for detecting meniscal tears showing sensitivities greater than $90 \%$ [11]. These results were in concordance with our results for both medial and lateral meniscal tears.

\section{Conclusion:}

The volumetric fat suppressed proton density sequence demonstrated total concordance with the standard 2D sequences in diagnosing both medial and lateral meniscal tears with the clear advantage of having shorter acquisition time.

\section{References}

1- CHEN W., ROSENBERG J.K., BEATTY P.J., KIJOWSKI R., HARGREAVES B.A., et al.: Optimizing isotropic three-dimensional fast spin-echo methods for imaging the knee. J. Magn. Reson. Imaging, 2014.
2- DAVIS K.W., BLANKENBAKER D.G., WOODS M.A., DEL RIO A.M. and De SMET A.A.: Evaluation of the menisci of the knee joint using three-dimensional isotropic resolution fast spin-echo imaging: Diagnostic performance in 250 patients with surgical correlation. Skeletal Radiol., 41 (2): 169-78. Crossref, Medline, 2012.

3- FRIEDRICH K.M., REITER G., KAISER B., et al.: Highresolution cartilage imaging of the knee at $3 \mathrm{~T}$ : Basic evaluation of modern isotropic 3D MR-sequences. Eur. J. Radiol., 78 (3): 398-405. Crossref, Medline, 2011.

4- JUNG J.Y., YOON Y.C., KWON J.W., et al.: Diagnosis of internal derangement of the knee at 3.0-T MR imaging: $3 \mathrm{D}$ isotropic intermediate-weighted versus $2 \mathrm{D}$ sequences. Radiology, 253: 780-7, 2014.

5- LEE S.Y. and PARK N.H.: Three-dimensional isotropic T2-weighted fast spin-echo (VISTA) knee MRI at 3.0 T in the evaluation of the anterior cruciate ligament injury with additional views: Comparison with two-dimensional fast spin-echo T2-weighted sequences. Acta Radiol. Nov., 57 (11): 1372-9, 2016.

6- WOODS M., Del RIO A.M., De SMET A.A. and REEDER S.B.: Clinical usefulness of adding 3D cartilage imaging sequences to a routine knee MR protocol. AJR Am. J. Roentgenol., 196 (1): 159-67. Crossref, Medline, 2011.

7- YOON Y.C., SEO J.M. and KWON J.W.: 3D isotropic turbo spin-echo intermediate-weighted sequence with refocusing control in knee imaging: Comparison study with 3D isotropic fast-field echo sequence. Acta Radiol., 52 (10): 1119-24. Crossref, Medline, 2011.

8- PASS B., ROBINSON P., HODGSON R. and GRAINGER A.J.: Can a single isotropic 3D fast spin echo sequence replace three-plane standard proton density fat-saturated knee MRI at 1.5 T? Br. J. Radiol., 88, 2015.

9- AI T., ZHANG W., PRIDDY N.K. and LI X.: Diagnostic performance of CUBE MRI sequences of the knee compared with conventional MRI. Clin. Radiol., 67: e58-63. doi: $10.1016,2012$

10- KUDO H., INAOKA T., KITAMURA N., NAKATSUKA T., KASUYA S., KASAI R., et al.: Clinical value of routine use of thin-section 3D MRI using 3D FSE sequences with a variable flip angle technique for internal derangements of the knee joint at 3T. Magn. Reson. Imaging, 31: 1309-17, 2013.

11-NANCY M. MAJOR and CLYDE A. HELMS: Comparison of 3D PDFS Versus Conventional Spin-Echo MRI for Evaluating Meniscal Tears American Journal of Roentgenology, 184: 1740-3, 2011. 


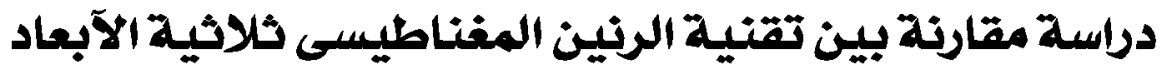

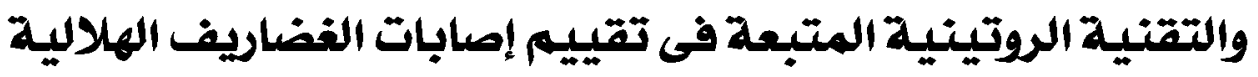

الهدف من هذه الدراسة هو تقييم دود الرنين المغناطيسى ثلاثية الآبعاد عالية التباين المرضى المشتبه بإصابتهم بقطع الغضاريف الهلالية. آجريت هذه الدراسة فى مستشفيات جامعة القاهرة على اله من المرضى. خضع كل مريض إلى تصوير كامل لمفصل الركبه بإستخدام الرنين المغناطيسى ثلاثية الآبعاد والتقنية الروتينية المتبعه. تم التاكد من تطابق عالى فى النتائج بين تقنية الرنين المغناطيسى ثلاثية الآبعاد والتقنية الروتينية فى الكشف عن إصابات الفضاريف الهلالية الداخلية والخارجية. 